\title{
NEW METHODS FOR COMPARATIVE RESEARCH?
}

\author{
Claude Rubinson and Charles C. Ragin
}

\section{INTRODUCTION}

Shalev's (2007) critique of the use of multiple regression in comparative research brings together and synthesizes a variety of previous critiques, ranging from those focusing on foundational issues (e.g., the persistent problem of limited diversity), to estimation issues (e.g., the unrealistic assumption of correct model specification), to narrow technical issues (e.g., the difficulty of deriving valid standard errors for regression coefficients in pooled cross-sectional time-series models). Broadly speaking, these concerns can be described as epistemological, theoretical, and methodological, respectively. While the distinctions among these three are not always clear-cut, the tripartite scheme provides a useful way to map the different kinds of critiques that may be directed at the use of regression analysis in comparative research. In the first half of this essay we build upon Shalev's discussion to clarify the conditions under which regression analysis may be epistemologically, theoretically, or methodologically inappropriate for comparative research. Our goal is to situate Shalev's specific critiques of the use of multiple regression in comparative work within the context of social research in general.

In the second half of this essay, we focus on Shalev's proposed solutions. We commend Shalev for offering constructive solutions to the problems he

Capitalisms Compared

Comparative Social Research, Volume 24, 373-389

Copyright (C) 2007 by Elsevier Ltd.

All rights of reproduction in any form reserved

ISSN: 0195-6310/doi:10.1016/S0195-6310(06)24013-4 
raises. Too often, critiques end without solutions being offered, demoralizing those who are committed to empirical research. However, we feel that Shalev has overlooked the fact that the issues he raises are addressed more completely and fully in the growing literature on Qualitative Comparative Analysis (hereafter, QCA) and fuzzy-set analysis. ${ }^{1}$ We argue that QCA and related methods both encompass and extend Shalev's proposed solutions and provide a strong foundation for systematic, case-oriented comparative research.

\section{THE CRITIQUE OF MULTIPLE REGRESSION}

One of the central themes of Shalev's critique of multiple regression is its incongruence with case-oriented analysis. Case-oriented approaches might be preferred for several reasons, in addition to the simple fact that comparativists tend to study small $N \mathrm{~s}$. For example, case-oriented methods are better suited for the types of questions that comparative researchers typically ask. Unlike multiple regression, case-oriented techniques such as QCA and fuzzy-set analysis are specifically designed to address questions about necessary or sufficient conditions that often motivate comparative research. Furthermore, case-oriented techniques can be used to address causal complexity. Finally, case-oriented methods such as QCA, fuzzy-set analysis, and those recommended by Shalev are more closely aligned with the epistemological orientations held by many comparative researchers. This orientation identifies the case - rather than the variable - as the fundamental unit of interest to social researchers.

\section{The Epistemological Critique}

The epistemological critique of the use of regression analysis in comparative research is straightforward: the method results in unproductive representations of social phenomena. Social research is best described as the construction of scientific representations of social life (Ragin, 1994). To the extent that applications of regression analysis result in representations that do not resonate with researchers' understandings, the method is called into question. The primary reason that these representations are found lacking is that case-oriented comparative researchers keep cases, not the net effects of variables, at the forefront of their analyses. It is not that case-oriented researchers dismiss variables, but rather that they perceive that it is not 
variables but cases that have relationships with one another. The variableoriented researcher shows, for example, that poverty is correlated with crime and that economic development is correlated with democracy. The caseoriented researcher observes that criminals tend to be poor, especially the ones that get caught, and that economically developed countries tend to be democratic. Although subtle, this distinction entails fundamentally different views of social phenomena. Where variable-oriented researchers view the social world as a manifestation of the myriad relationships among variables, case-oriented researchers see many different kinds or sets of cases.

For the case-oriented researcher, the problem with regression analysis is that it veils cases. Regression analysis describes the relationships between independent and dependent variables which, from the vantage of caseoriented research, is a limited and fragmented picture of reality. Note that this is not a technical critique of the method's capabilities but, rather, a reaction to the world-view inherent in the method. In regression analysis, cases do not constitute anything in and of themselves; they are merely carriers of information about the relationships among variables. ${ }^{2}$ The case-oriented researcher, however, requires methods that maintain the constitution and integrity of the cases under observation. Stated simply, regression is incapable of doing this and, therefore, is an inadequate platform for conducting caseoriented research. Although strongly worded, such a statement should not be controversial. It is not a deficiency of regression that it fails to meet the needs of case-oriented researchers but simply reflects the fact that it meets the needs of variable-oriented researchers so well. Its strength is also its weakness. While comparative researchers tend to be case-oriented, this coupling is not mandatory. Regression analysis is perfectly suitable in the hands comparative researchers who see cases as instances of relationships between variables.

\section{The Theoretical Critique}

Regression analysis is best at answering theoretically framed questions about the net effects of competing independent variables on a dependent variable (Ragin, 2006). In a multiple regression model, it is assumed that any single variable is sufficient by itself for achieving an impact on the outcome and that no variable is necessary. Thus, regression analysis is not well-suited for the analysis of causal complexity. As Shalev (2007) notes: "The results will be ambiguous because they will be unable to distinguish between additive effects, conditional effects, and multiple causal pathways." These

limitations are especially problematic when comparative researchers attempt 
to use regression to answer theoretical questions that it is not designed to answer. Unfortunately, such attempts are far too common. Shalev provides an example in his review of Rothstein's analysis of union membership. Rothstein hypothesizes, in essence, that the presence of the Ghent system is a necessary condition for high levels of unionization. However, he attempts to test this hypothesis by regressing percent union on presence of Ghent, strength of left government, and potential union membership. While a statistically insignificant effect for Ghent might undermine the hypothesis, Shalev (2007) points out that "the regression could not make a positive case for Rothstein's argument." Regression coefficients report the partialed effects of each independent variable on the dependent variable. There is no basis in this type of analysis for privileging the effect of Ghent as a necessary condition for high levels of unionization.

Sometimes it is difficult to correctly identify the method most appropriate for answering a given theoretical question. Consider another study that Shalev reviews, Hall and Franzese's (1998) investigation of the effect of central bank independence on unemployment rates. Shalev (2007) identifies two central hypotheses:

In nations where wage coordination is high, an increase in the independence of the central bank is associated with a very small increase in the rate of unemployment.

Where wage coordination is low, however, an increase in the independence of the central bank is associated with a substantial increase in the rate of unemployment.

These hypotheses encompass both case-oriented and variable-oriented questions. On the one hand, Hall and Franzese are asking about nations with high versus low levels of wage coordination, a question that is case-oriented in nature. On the other hand, they are asking about the association between central bank independence and unemployment rates, a question that is variable-oriented in nature. This disjuncture results from Hall and Franzese's recognition of the contextual effect of wage coordination. In regression analysis, contextual effects are operationalized through interaction terms. Indeed, from a variable-oriented perspective, the two hypotheses are one. It would be more precise to test the single hypothesis that "as wage coordination decreases, the strength of the positive association between central bank independence and the unemployment rate increases," and, in fact, this is the hypothesis that they test, using an interaction term (Hall \& Franzese, 1998, p. 519). Dichotomizing the wage coordination measure permits Hall and Franzese to interpret their results as applying to nations with high versus low levels of wage coordination. Strictly speaking, however, their results only describe relationships among variables. 
Case-oriented techniques, by contrast, downplay the relationships among variables and instead emphasize how the countries in the data set constitute 18 combinations of the variables of interest. Shalev (2007) presents a rudimentary configurational view in his Chart 2. Examining Chart 2, what stands out is not the relationship between unemployment rates, central bank independence, and wage coordination but that, with the exception of Australia, all of the states with relatively low levels of wage coordination also have relatively high rates of unemployment. Furthermore, again with the exception of Australia, all countries with relatively low rates of unemployment have relatively high levels of wage coordination. Setting Australia aside, these results indicate that having high levels of wage coordination is a necessary condition for low levels of unemployment and, correspondingly, that low levels of wage coordination are sufficient for high rates of unemployment. Such conclusions naturally lead to a focus on the anomalous case of Australia: why is Australia's unemployment rate so much lower than one would otherwise expect? How does Australia differ from France, which has similar scores on wage coordination and central bank independence? Similar questions are raised regarding Germany, Denmark, Finland, and Norway: these countries all have high levels of wage coordination; why do they also have high unemployment rates? In short, the use of caseoriented techniques disposes the researcher to focus on the characteristics of the cases under investigation rather than the relationships among the variables.

\section{The Methodological Critique}

The most well-known critique of the use of regression analysis in comparative research is methodological, the so-called "small- $N$ problem." Since comparative researchers generally study only a handful of cases, sophisticated regression techniques quickly exhaust available degrees of freedom. Comparative researchers often emphasize that case-oriented analytic techniques can better address issues of causal complexity than variable-oriented analytic techniques (see, e.g., Rueschemeyer \& Stephens, 1997); however, Shalev (2007) correctly points out that:

The problem is not that MR does not have or could not invent technologies for dealing with such complexities. Nonlinear functional forms, interaction effects and (in timeseries analysis) complex lag structures immediately come to mind. The point is that because such techniques are either difficult to employ or impose a steep statistical penalty due to the "small n problem," they are rarely or insufficiently used. 
To the extent that regression analysis is ill-suited for comparative analysis due to limited degrees of freedom, then, there are three possible solutions. One is to increase the number of observations available for analysis through additional data collection or a reformulation of the research question (see King, Keohane, \& Verba, 1994). A second is to use a technique such as factor analysis or metric scaling to reduce the dimensionality of the model's vector space. The third is to turn to case-oriented analytic techniques that are not directly constrained by considerations of degrees of freedom.

Pooled cross-sectional designs are a common example of the first option. Shalev (2007) cogently describes the methodological problems that can accompany this technique, noting that such designs reflect the limitations of both cross-sectional and longitudinal studies: "pooled designs are the worst of both worlds." Rather than artificially increasing the number of observations by using pooled designs, Shalev recommends reducing the number of causal variables, as illustrated in his discussion of Esping-Andersen's analysis of welfare regimes. Reduction of vector space dimensionality is a long standing recommendation (Berg-Schlosser \& De Meur, 1997). However, comparative researchers should be cognizant of the limitations of data reduction techniques such as factor analysis. Specifically, in order to create an index from several causal conditions factor analysis rescales correlated conditions and then sums their scores. The assumption, in effect, is that the different conditions that go into an index are partially substitutable such that any one condition may compensate for any other condition. Thus, factor analysis, like regression analysis, masks causal complexity and veils case specificity. The application of vector reduction techniques such as factor analysis and metric scaling demands theoretical justification; they should not be used as easy, technical solutions to problems associated with limited degrees of freedom. This leaves the third solution to the degrees-of-freedom shortage, which is to use case-oriented methods such as QCA, fuzzy-set analysis, or the methods Shalev proposes. While attractive, this path is not necessarily a panacea, for it carries with it a world-view that emphasizes similarities and differences among cases, not relationships among variables.

\section{Discussion}

Shalev reviews a number of arguments as to why regression analysis is often inappropriate for comparative research. The methodological critique is most commonly made, and it is easiest to understand: Comparative researchers frequently study a limited number of cases; under such conditions, the 
assumptions of regression analysis are very difficult to meet. The theoretical and epistemological critiques are less frequently made, but are more important because they are directed at more fundamental concerns. The theoretical critique observes that regression analysis is best-suited for answering only certain types of questions regarding relationships among variables. Frequently, however, these are not the types of questions that interest comparative researchers. The epistemological critique observes that regression analysis carries with it a variable-oriented world-view that is incongruent with the case-oriented world-view, which is common, though certainly not universal, among comparative researchers. Taken together, these three critiques point to the need for methods that meet the specific requirements epistemological, theoretical, and methodological - of case-oriented comparative researchers.

Shalev (2007) writes "MR remains by far the predominant mode of numerical data analysis and most of its critics sees qualitative analysis (whether formal or not) as the only real alternative. This paper seeks to promote a third way." We share Shalev's concern. The conventional division between quantitative and qualitative research techniques tends to hinder - rather than benefit - social research by implicitly limiting case-oriented researchers to qualitative tools and variable-oriented researchers to quantitative tools.

\section{ALTERNATIVES TO REGRESSION ANALYSIS}

Shalev largely neglects QCA and fuzzy-set analysis in favor of his proposed methods. In the second half of this essay, we contest this oversight. QCA and fuzzy-set analysis are, in fact, more elaborate and refined versions of the methods Shalev recommends.

\section{Shalev's Critique of QCA}

Shalev (2007) suggests that, with regard to comparative research, QCA and fuzzy-set analysis suffer some of the same shortcomings as regression analysis:

Ragin's methods are not "qualitative" in the sense of relying on the interpretive skills of analysts wading knee-deep in thick description. If anything, as Griffin and Ragin (1994, p. 10) have insisted, QCA is more like MR: both apply rules that are independent of the researcher, and both treat cases as "discrete, multiple instances of more general phenomena." 
It is true that QCA shares a few characteristics with regression analysis. Both are formal methods and, as such, are characterized by the application of procedures that are independent of the researcher. But whereas regression is an application of linear algebra rooted in matrix theory (Marcus \& Minc, 1988), QCA and fuzzy-set analysis are applications of set theory (Whitesitt, 1995). Set theory is used, very simply, to formalize the logic of comparative analysis, as practiced by case-oriented researchers. The primary goal of the formal procedures implemented in QCA is to prevent researchers from drawing illogical conclusions from comparative evidence, especially when the $N$ of cases is more than a handful. Consider the truth table, which forms the foundation of both QCA and fuzzy-set analysis. Superficially, it appears similar to a conventional data set in that it utilizes a "cases-by-variables" format. But the rows of a truth table are not observations as they are in a conventional data set. Rather, each row represents a logically possible combination of causal conditions. ${ }^{3}$ It is up to the researcher to determine which of these combinations map onto real-world cases. Frequently, the process of mapping the causal configurations onto real-world cases will prompt researchers to revisit and revise their classification schema, based on in-depth analysis of cases. From the same Griffin and Ragin (1994, p. 10) article:

To resolve the contradictions ${ }^{4}$ in their data, the authors intensively reexamined both the configurations producing contradictory outcomes and the cases in those configurations. They searched for errors in their original classification, thought more deeply about whether their dichotomous measure of labor management practices was too crude, looked anew at their interviews with personnel managers, and strategically compared mills in contradictory configurations with mills in configurations free of contradictory outcomes. All of this interpretive work on classification - really, on the meaning of their outcome factor and its applicability to several of their cases - was but a prelude to their explanatory analysis.

It is through the construction, revision, and refinement of truth tables that QCA and fuzzy-set analysis rely "on the interpretive skills of analysts wading knee-deep in thick description" (see also Ragin \& Rihoux, 2004 and the commentary and exchanges it generated in a special issue of Qualitative Methods devoted to QCA; see also the three-way exchange on QCA versus regression analysis published in Studies in Comparative International Development: Achen, 2005; Seawright, 2005; Ragin, 2005). As with the alternative techniques that Shalev proposes, effective application of QCA and fuzzy-set analysis depends directly upon the researcher's substantive knowledge of the cases under investigation. 


\section{Shalev's Tabular Technique and QCA}

Shalev's first proposed alternative to regression analysis makes use of tabular techniques. Reanalyzing Rothstein's model of union membership, Shalev clusters countries according to the different combinations of causal conditions that they exhibit. This technique has two benefits. First, it provides a direct test of a hypothesis that conventional regression analysis could not provide, that is, Rothstein's hypothesis that "the highest levels of unionization have been reached only in countries where [the Ghent system] is in place" (Shalev, 2007). Shalev's tabular technique clearly identifies the combinations of conditions linked to high rates of unionization, confirming Rothstein's hypothesis. The second benefit of Shalev's (2007) tabular technique is that, in moving the individual countries to the forefront of the investigation, it also "point[s] the interested researcher to the most fertile questions for selective case comparisons." Shalev's tabular technique leads naturally to the investigation of similarities and differences among cases in a way that analysis of regression residuals does not.

The insight underlying Shalev's tabular technique - that it is combinations of conditions that matter - is the same insight that underlies QCA (Ragin, 1987). QCA is built around the analysis of a "truth table" that delineates the various combinations of conditions linked to the presence/ absence of an outcome. As with Shalev's tabular technique, QCA permits the investigation of necessary and sufficient conditions and keeps the individual cases at the forefront of the analysis. QCA has a number of advantages over Shalev's tabular technique. The most obvious advantage is conciseness. As the number of causal conditions increases, tabular analysis quickly becomes unmanageable. A truth table, however, can accommodate a large number of causal conditions. Furthermore, the existence of truth table reduction algorithms provided in software packages such as fs/QCA simplifies the accompanying analysis. Shalev (2007) notes that determining the proper setup of the table "requires some forethought" due to the complexity of the analysis. Researchers using QCA need only define and measure the relevant causal conditions. The method's algorithm identifies the causal configuration(s) linked to the outcome under investigation.

Shalev emphasizes that an advantage of his tabular technique over regression analysis is that it places cases in the foreground of the analysis. QCA does this as well. Using either technique, researchers will not simply determine that high union membership is present in countries with a combination of small size, medium or strong left parties, and the presence of the Ghent system; they can explore additional avenues of investigation: "In 
particular, it must be questioned whether the Ghent system alone can explain the very large differences in density between otherwise well-matched countries: Belgium versus the Netherlands, and Sweden and Denmark versus Norway" (Shalev, 2007). But QCA has an additional advantage in that it forces investigators to resolve contradictions (cases with similar causal configurations that produce divergent outcomes). For example, Shalev simply ignores the contradiction between Switzerland and Ireland: both are small countries with weak left parties and no Ghent system, but union membership is weak in Switzerland and strong in Ireland. QCA forces the researcher to confront such contradictions and decide how to deal with them (see Ragin \& Rihoux, 2004; Ragin, 2005). In this way, QCA structures a close interaction between researcher and cases.

Another advantage of QCA over Shalev's tabular technique regards the analysis of counterfactual cases. Shalev (2007) correctly points out that the social world is characterized by limited diversity:

In cross-national quantitative research the situation is very different [than in survey research]. We often analyze the entire universe of cases, and if not it is usually because of lack of data rather than sampling considerations. For the most part then, if a particular configuration of attributes does not exist in a cross-national data set, it does not exist at all (emphasis in original).

Shalev (2007) raises the issue of limited diversity within his critique of regression analysis and comments that "it cannot be denied that one of the tests of a useful causal model is that it be capable of answering counterfactual questions." QCA provides just such a capability, for the analysis of limited diversity is a long-standing focus of the approach (see Ragin, 1987). As detailed in Ragin and Sonnett (2004), QCA includes tools especially designed for the analysis of "remainder" causal combinations (that is, logically possible combinations of conditions that lack empirical instances). Such analyses formalize the thought experiments proposed by Weber (1905) by treating the remainder combinations as counterfactual cases. By incorporating the analysis of remainders into QCA, the researcher can better assess the causal role that specific conditions play in bringing about the outcome in question.

\section{Shalev's Three-Dimensional Plots and Fuzzy-Set Analysis}

A second technique that Shalev utilizes in his reanalyses of Hall and Franzese (1998) and Garrett (1998) is that of three-dimensional scatterplots, with the third dimension represented as proportionately sized "bubbles." 
These scatterplots can be seen as nascent fuzzy-sets. Reanalyzing Garrett's data, Shalev (Chart 4) clusters countries according to their degree of capital restriction and trade openness. In set-theoretic terms, these clusters represent subsets. Shalev identifies three subsets: a set of countries with low levels of both capital restriction and trade openness, ${ }^{5}$ a set of countries with high levels of capital restriction and middling levels of trade openness, and a set of countries with low levels of capital restriction and high levels of trade openness. Shalev observes that the countries in Garrett's analysis exhibit limited diversity: there are specific regions of the property space that are void of cases. In particular, he notes that there are no cases for the combinations of (a) high left-labor power with low capital restriction or (b) low left-labor power with high trade openness. As Shalev notes, Garrett conducted tabular analyses that included precisely these combinations.

Shalev's critique of Garrett reflects the distinctive manner by which comparative researchers often measure their variables. Garrett (1998, p. 84) employs relative measures of left-labor power, capital restriction, and trade openness: "Low (high) levels of trade and capital mobility refer to the 20th (80th) percentile scores in the sample. Low (high) levels of left-labor power refer to the 20th (80th) percentile scores on left-labor power index." For Garrett, scores are low or high relative to the median; indeed, it would be more accurate to use the labels "lower" and "higher" to reflect this operationalization. For comparative researchers, adjectives such as "low" and "high" generally describe qualitative conditions measured against a defined standard. Consider the set of Western European countries. Although there is certainly variation in GDP per capita among these countries, all may reasonably be considered rich - depending upon how the researcher defines "rich." Case-oriented methods do not evaluate variation in the same way that variable-oriented methods do. In case-oriented research, it is the substantive meaning of the scores that is most important; scores must be calibrated relative to some standard, not simply relative to a measure of central tendency. In qualitative work, measurement is an interpretive process, based on the researcher's theoretical and substantive knowledge.

From this viewpoint, Shalev makes the same general error as Garrett. When constructing his scatterplots, Shalev does not consider the substantive meaning of the various scores but simply accepts them at face value. Examining Chart 4, for example, Shalev (2007) identifies a cluster of social democracies consisting of Sweden, Denmark, Austria, and Norway. Finland is not included in this cluster, presumably due to its higher level of capital restriction. But does Finland's exclusion make sense? Garrett's measure of capital restriction is simply a count of four types of government restrictions 
on capital mobility. Excluding Finland from the social-democratic cluster assumes that the raw number of restrictions matters. It is not clear that this is true. For example, in an investigation of foreign exchange market turbulence, Eichengreen, Andrew, and Wyplosz (1995) operationalize capital restriction simply as a dummy variable indicating the presence or absence of any capital controls. This operationalization indicates that the researchers believe that capital restrictions are substitutable for one another and, furthermore, that their effects are not necessarily additive. It may be that the difference between Finland's level of capital restriction and those of Sweden, Denmark, Austria, and Norway amounts to nothing more than irrelevant variation, and Finland should be included in the social-democratic cluster.

Shalev's reanalysis of Hall and Franzese (1998, Chart 2) displays the same shortcoming. Hall and Franzese do not justify the dichotomization of their institutional variables, and Shalev appropriately criticizes this oversight. But it is by no means clear that Shalev's strategy of disaggregating the variables is better. Both actions are arbitrary. Shalev's approach assumes that the data - and the variation in the data - speak for themselves. But researchers must always interpret scores and evaluate what they mean. Because Shalev does not find a pattern in Chart 2, he concludes that Hall and Franzese's findings are an artifact of their dichotomizing their measures. But it is also possible that Shalev's lack of findings is a result of his failure to properly calibrate his measures, using theoretical and substantive knowledge to guide the interpretation of scores.

Fuzzy-set analysis forces researchers to calibrate their measures carefully; the resulting fuzzy membership scores must be substantively meaningful. In fuzzy-set analysis, scores indicate the degree of membership of cases in a given set. A country may be classified as fully, partially, or not belonging to the set of countries with, for example, high left-labor power or high capital restriction. ${ }^{6}$ After the researcher calibrates membership scores, formal fuzzy-set techniques can be applied to determine the subset relationships that exist among the cases. Shalev derives his clusters using ad hoc procedures; fuzzy-set analysis applies set theory to the same end, based on the researcher's interpretation of each case's degree of membership in the relevant sets.

A further difference between Shalev's clustering technique and fuzzy-set analysis concerns the role that the derived subsets play in the subsequent analysis. Shalev's clusters are primarily descriptive. By keeping the cases in the foreground of his reanalysis of Garrett, Shalev's technique permits him to distinguish a social-democratic subset, an autarchic subset, and a 
small-state subset. In fuzzy-set analysis, however, subsets are not merely descriptive but also provide a foundation for the analysis of causality. Through the application of set theory and fuzzy algebra, fuzzy-set analysis provides formal methods for evaluating necessary and sufficient conditions.

Fuzzy-set analysis is a variant of QCA; as such, it shares QCA's advantages. Like QCA, fuzzy-set analysis can accommodate a substantial number of causal conditions. Shalev's scatterplots are useful, but it is difficult to visualize a plot with more than three dimensions. Reflecting the fact that his technique grants explanatory primacy to just two dimensions at a time, Shalev is unable to incorporate level of left-labor power into his clusters. Fuzzy-set analysis, on the other hand, locates each case's position in a vector space with a much larger number of dimensions. (In practice, most researchers use from four to nine.) Also like QCA, fuzzy-set analysis makes use of truth tables and provides formal techniques for identifying the various causal configurations linked to the outcome under investigation and for the analysis of counterfactuals.

\section{Discussion}

Case-oriented comparative researchers seek explanation by exploring the similarities and differences among cases. The problem with variableoriented techniques such as multiple regression is that they render cases invisible. At the heart of Shalev's tabular and scatterplot techniques is an attempt to bring cases to the foreground of the analysis in order to facilitate the researcher's case-oriented analysis. We are surprised that Shalev positions his techniques as alternatives to QCA and fuzzy-set analysis when they are in fact rudimentary versions of QCA and fuzzy-set analysis. Perhaps the formality of QCA and fuzzy-set analysis makes these techniques appear inappropriate for case-oriented research. With regard to formal quantitative methods, Shalev (2007) cautions against such a reaction: "provided they fit researchers' theoretical assumptions, there is no reason why inductive multivariate statistical methods should not be exploited by comparativists." We extend this astute guidance to formal qualitative methods. It would be unfortunate if comparative researchers dismissed QCA and fuzzy-set analysis simply due to their formality.

As formal methods, QCA and fuzzy-set analysis provide useful ways of simplifying many of the common tasks that comparative researchers face. In constructing the tabular presentation of Rothstein's data, Shalev faced two tasks: developing the measures of the various causal conditions and building 
a useful table showing key patterns. QCA formalizes the latter task, freeing researchers to concentrate on the former. Similarly, in developing his scatterplot of Garrett's data, Shalev had to measure the various indicators, build the scatterplot, and identify the relevant subsets. Fuzzy-set analysis frees researchers to concentrate on the measurement and calibration of set memberships; set-theoretic analysis of configurations of set memberships is accomplished using software. Shalev suggests that QCA and fuzzy-set analysis distance comparative researchers from their cases; in fact, the opposite is true. By formalizing the most difficult analytic tasks involved in comparative research - the comparison of cases as configurations of similarities and differences - these methods free researchers to direct their time and energy toward getting to know their cases well.

QCA and fuzzy-set analysis enhance comparative research by facilitating case comparisons. The analytic process brings contradictions to light and reveals conditions of limited diversity, providing avenues for further study. As noted above, QCA also offers procedures for the consideration of counterfactual cases. Perhaps most important, QCA and fuzzy-set analysis provide methods for the analysis of necessary and sufficient conditions as well as multiple conjunctural causation. These procedures, while formal, remain under the control of the researcher. In this manner, QCA and fuzzy-set analysis offers the transparency desired by comparative researchers while remaining faithful to the theoretical and substantive expertise of the researcher.

\section{CONCLUSION}

Michael Shalev's essay is an important contribution to the continuing debate on appropriate methods for comparative research. Drawing upon previously published research, he demonstrates a variety of ways in which the inappropriate application of multiple regression has compromised comparative work. Shalev proposes a number of alternative research strategies better suited to the needs of case-oriented researchers. It is important to note that Shalev's recommendation is not that comparative researchers abandon regression analysis or quantitative methods altogether, but instead that they learn to better match research questions and techniques. We strongly endorse this recommendation.

In the first half of this essay we clarify the various ways in which the choice of method matters. Different research methods embody different epistemological world-views. These different world-views shape the 
questions that scholars may ask and, consequently, their results. Changing the research technique, then, can fundamentally alter the research project. In the second half of the essay, we address Shalev's critique of QCA and fuzzyset analysis. Contrary to Shalev's assessment, QCA and fuzzy-set analysis are case-oriented techniques finely tuned to the needs and practices of comparative researchers. We demonstrate that QCA and fuzzy-set analysis incorporate and extend the insights and techniques of Shalev's recommended methods. Although Shalev positions his methods as alternatives to QCA and fuzzy-set analysis, we find greater similarity than difference among the approaches.

Comparative researchers frequently find themselves in the gulf between small- $N$ qualitative studies and large- $N$ quantitative studies (Ragin, 2000). Most of the studies that Shalev reviews involve between 14 and 18 countries, numbers small enough to constrain the available degrees of freedom but large enough to hinder in-depth analysis of each case. Case-oriented techniques such as QCA, fuzzy-set analysis, and those developed by Shalev permit the pursuit of both breadth and depth of understanding by assisting comparative researchers in their search for commonalities and differences across cases.

\section{NOTES}

1. See, for example, the extensive international bibliography on comparative methodology, QCA, and fuzzy sets at www.compasss.org, which lists more than 250 applications of QCA.

2. It is important to note that this critique does not apply to all quantitative methods. Social network analysis, for example, is both quantitative and caseoriented. Network analytic methods can be used to describe not only the cases within a network but also the overall network (the network itself, constituting a case). Reflecting the case-oriented researcher's concern with the relationships among sets, methods exist to assess the intersections, unions, and divisions within and between social networks. The point here is simply that one should not assume that caseoriented research is necessarily qualitative. Likewise, there is no reason to assume that variable-oriented research is necessarily quantitative.

3. In the article that Shalev references, Griffin and Ragin (1994, p. 10) overstate the resemblance between regression and QCA when they write "So similar are QCA and logit regression in causal epistemology, for example, that the very same data matrix can serve both kinds of analyses." Logit regression would be applied directly to the data set; QCA (or fuzzy-set analysis) would be applied to a truth table derived from the data set. Popular software applications such as fs/QCA automate the transformation of a conventional data set into a truth table, further obscuring this distinction. 
4. A "contradiction" occurs when there are cases with identical causal configurations, except that some of the cases exhibit the outcome under investigation and others do not. Notice how the problem of contradictions highlights the difference between a truth table (in which rows represent configurations of causal conditions) and a conventional data set (in which rows represent observations).

5. The text indicates that this subset includes seven countries but only six are presented. We assume that France - which was included in Garrett's original analysis - was inadvertently omitted from this subset and would not change the results of the analysis.

6. Fuzzy scores range between 0.0 and 1.0. A score of 0.0 indicates that a case is fully out of the set of interest while a score of 1.0 indicates that a case is fully in the set. Scores between 0.0 and 0.5 indicate that a case is "more out than in" while scores between 0.5 and 1.0 indicate that a case is "more in than out."

\section{REFERENCES}

Achen, C. H. (2005). Two cheers for Charles Ragin. Studies in Comparative International Development, 40(1), 27-32.

Berg-Schlosser, D., \& De Meur, G. (1997). Reduction of complexity for a small-N analysis: A stepwise multi-methodological approach. Comparative Social Research, 16, 133-162.

Eichengreen, B., Andrew, K. R., \& Wyplosz, C. (1995). Exchange market mayhem: The antecedents and aftermath of speculative attacks. Economic Policy, 10, 251-312.

Garrett, G. (1998). Partisan politics in the global economy. Cambridge: Cambridge University Press.

Griffin, L., \& Ragin, C. C. (1994). Some observations on formal methods of qualitative analysis. Sociological Methods \& Research, 23, 4-21.

Hall, P. A., \& Franzese, R. J., Jr. (1998). Mixed signals: Central bank independence, coordinated wage bargaining, and European Monetary Union. International Organization, 5 , $505-535$.

King, G., Keohane, R. O., \& Verba, S. (1994). Designing social inquiry: Scientific inference in qualitative research. Princeton, NJ: Princeton University Press.

Marcus, M., \& Minc, H. (1988). Introduction to linear algebra. New York: Dover.

Ragin, C. C. (1987). The comparative method: Moving beyond qualitative and quantitative strategies. Berkeley: University of California Press.

Ragin, C. C. (1994). Constructing social research. Thousand Oaks, CA: Pine Forge Press.

Ragin, C. C. (2000). Fuzzy-set social science. Chicago: The University of Chicago Press.

Ragin, C. C. (2005). From fuzzy sets to crisp truth tables. http://www.compasss.org/ Raginfztt_April05.pdf

Ragin, C. C. (2006). The limitations of net-effects thinking. In: B. Rihoux \& H. Grimm (Eds), Innovative comparative methods for policy analysis (pp. 13-42). New York: Springer.

Ragin, C. C., \& Rihoux, B. (2004). Qualitative comparative analysis: State of the art and prospects. Qualitative Methods, 2(2), 3-13.

Ragin, C. C., \& Sonnett, J. (2004). Between complexity and parsimony: Limited diversity, counterfactual cases, and comparative analysis. In: S. Kropp \& M. Minkenberg (Eds), Vergleichen in der Politikwissenschaft. Wiesbaden: VS Verlag für Sozialwissenschaften. 
Rueschemeyer, D., \& Stephens, J. D. (1997). Comparing historical sequences - a powerful tool for causal analysis. Reply to John Goldthorpe's current issues in comparative macrosociology. Comparative Social Research, 16, 55-72.

Seawright, J. (2005). Qualitative comparative analysis vis-a-vis regression. Studies in Comparative International Development, 40(1), 3-26.

Shalev, M. (2007). Limits and alternatives to multiple regression in comparative research. Comparative Social Research, 24, 259-308.

Weber, M. (1905). Objective possibility and adequate causation in historical explanation. In: E. A. Shils \& H. A. Finch (Eds), The methodology of the social sciences (pp. 164-188). Glencoe, IL: The Free Press.

Whitesitt, J. E. (1995). Boolean algebra and its applications. New York: Dover. 